\title{
Inhibitory effect of Angelica gigas on cold-induced RhoA activation in vascular cells
}

\author{
KANGWOOK LEE $^{1 *}$, MIN SOO CHAE ${ }^{2 *}$, SUNG-GOOK CHO $^{3}, \mathrm{HO} \mathrm{YEON} \mathrm{GO}^{4}$, SEUNG-HO SUN ${ }^{5}$, \\ JUNBOCK JANG ${ }^{6}$, KI-YONG JUNG ${ }^{7}$, YOU-KYUNG CHOI ${ }^{7}$, YUN-KYUNG SONG ${ }^{8}$, SUNG YONG SIM $^{9}$, \\ HYE LIM LEE ${ }^{10}$, MI SUK KANG ${ }^{11}$, CHAN-YONG JEON ${ }^{7}$ and SEONG GYU KO ${ }^{12}$
}

Departments of ${ }^{1}$ Science in Korean Medicine and ${ }^{2}$ Korean Medicine Obstetrics \& Gynecology, Graduate School, Kyung Hee University, Seoul 02477; ${ }^{3}$ Department of Biotechnology, Korea National University of Transportation, Cheongju, Chungbuk 27469; ${ }^{4}$ Department of Korean Internal Medicine, College of Korean Medicine, Semyung University, Chungju, Chungbuk 27136; ${ }^{5}$ Department of Oriental Internal Medicine, College of Korean Medicine, Sangji University, Wonju, Gangwon 23339; ${ }^{6}$ Department of Korean Medicine Obstetrics and Gynecology, College of Korean Medicine, Kyung Hee University, Seoul 02477; ${ }^{7}$ Department of Korean Internal Medicine, College of Korean Medicine, Gachon University, Seongnam, Gyeonggi 13120; Departments of ${ }^{8}$ Korean Rehabilitation Medicine, ${ }^{9}$ Korean Ophthalmology \& Otolaryngology, ${ }^{10}$ Korean Pediatrics and ${ }^{11}$ Acupuncture and Moxibustion Medicine, College of Korean Medicine, Gachon University, Seongnam, Gyeonggi 461701; ${ }^{12}$ Department of Preventive Medicine, College of Korean Medicine, Kyung Hee University, Seoul 02453, Republic of Korea

Received March 21, 2016; Accepted February 15, 2017

DOI: $10.3892 / \mathrm{mmr} .2017 .6404$

\begin{abstract}
The herbal extract Angelica gigas (AG) has been applied as a vasodilating agent for patients suffering from vascular diseases for many years; however, the underlying mechanism has not been fully elucidated. The present study hypothesized that the anti-vasoconstrictive effect of AG may be effective in the treatment of abnormal cold-mediated vasospasms that occur in Raynaud's phenomenon (RP). The effect of AG on the activity of ras homolog gene family member A (RhoA) was investigated in cold-exposed vascular cells. Vascular cells were pretreated to $\mathrm{AG}$, followed by a warm $\left(37^{\circ} \mathrm{C}\right)$ or cold $\left(25^{\circ} \mathrm{C}\right)$ incubation for $30 \mathrm{~min}$ and investigated with western blotting, ELISA and confocal microscopy. Cold treatment induced the activation of RhoA in pericytes and vascular endothelial cells, however this was reduced by treatment with AG. Furthermore,
\end{abstract}

Correspondence to: Professor Chan-Yong Jeon, Department of Korean Internal Medicine, College of Korean Medicine, Gachon University, 1342 Seongnam-daero, Sujeong-gu, Seongnam, Gyeonggi 13120, Republic of Korea

E-mail: joncy@gachon.ac.kr

Professor Seong Gyu Ko, Department of Preventive Medicine, College of Korean Medicine, Kyung Hee University, 3-10 Kyungheedae-ro 6-gil, Dongdaemun-gu, Seoul 02453, Republic of Korea

E-mail: epiko@khu.ac.kr

${ }^{*}$ Contributed equally

Key words: Raynaud's phenomenon, cold, RhoA, endothelin-1, Angelica gigas
AG treatment reduced the endothelin-1 (ET-1)-mediated RhoA activation in pericytes; however, cold-induced ET-1 production by vascular endothelial cells was not affected by treatment with AG. In addition, AG treatment suppressed the formation of stress fibers and focal adhesion complexes, and the cold-induced phosphorylation of focal adhesion kinase, proto-oncogene tyrosine-protein kinase Src and extracellular signal-related kinase. Therefore, AG treatment demonstrated an ability to reduce cold-induced RhoA activation in pericytes and vascular endothelial cells, and attenuated ET-1-mediated RhoA activation in pericytes. In conclusion, the present study indicated that AG may be useful for the treatment of RP.

\section{Introduction}

Raynaud's phenomenon (RP) is characterized by transient vasospasms within the fingers and/or toes, under cold or emotional stress conditions (1). RP is clinically classified into primary and secondary subtypes $(2,3)$, however the pathogenesis is not fully understood. Whereas primary RP does not reflect any other disorders, secondary RP is closely associated with life-threatening morbidities, including autoimmune disease or scleroderma (1). The cold-induced molecular mechanisms in vascular cells remain to be fully elucidated, however, previous research has indicated that cold-mediated vasoconstriction is regulated by ras homolog gene family member A (RhoA) (4-6). In endothelial cells (ECs), cold-induced RhoA activation increases the production of endothlein-1 (ET-1), a key vasoconstrictor in RP (7-9). Paracrine release of ET-1 from ECs activates RhoA in vascular smooth muscle cells (VSMCs) and pericytes (9-11). This RhoA activation induces the vasoconstriction of VSMCs $(4,6)$. Therefore, targeting RhoA may be an effective strategy for treatment of RP. 
Previous studies have investigated the biological and chemical efficacy of herbal medicines for the treatment of RP (4,12-16). Angelica gigas (AG) is currently used for the management of vascular diseases, including menopausal symptoms (17,18), atherosclerosis (19) and brain ischemia (20). The anti-vasoconstrictive effect of AG has been demonstrated in vitro and in vivo $(21,22)$, suggesting that $A G$ may be useful in managing the vascular dysfunction observed in RP. However, the effect of AG on cold-induced VSMC responses has not been studied.

The present study evaluated the inhibitory effect of AG on cold-induced vascular cell contraction. AG treatment inhibited cold- and ET-1-mediated RhoA activation in both pericytes and ECs. However, AG treatment had no effect on cold-induced ET-1 production in ECs. These results suggest that AG may be beneficial for relieving cold-induced vasoconstriction in RP, via the inhibition of RhoA.

\section{Materials and methods}

AG preparation and cell culture. AG was purchased from Hanpoong Pharm and Foods Company (Jeonju, Korea). $\mathrm{AG}$ roots were ground and subsequently extracted with $30 \%$ ethanol. The freeze-dried mixture was stored at $-80^{\circ} \mathrm{C}$. Human umbilical vein endothelial cells (HUVECs) were gifted by Kwang Seok Kim at Korea Institute of Radiological and Medical Science (Seoul, Korea). Human brain microvascular pericytes were purchased from ScienCell Research Laboratories, Inc. (1200; Carlsbad, CA, USA). HUVECs were cultured in endothelial medium supplemented with $5 \%$ fetal bovine serum (FBS) and 1\% endothelial cell growth supplement, microvascular pericytes were cultured in pericyte medium supplemented with 5\% FBS, $1 \%$ pericyte growth supplement, and $1 \%$ penicillin/streptomycin solution. FBS, growth supplements and media were purchased from ScienCell Research Laboratories, Inc. (Carlsbad, CA, USA). Cells were cultured at humidified incubator $\left(5 \% \mathrm{CO}_{2} ; 95 \%\right.$ relative humidity) at $37^{\circ} \mathrm{C}$.

Western blot analysis. To investigate ET-1-mediated RhoA activation, pericytes were incubated with 100 nM/1 ET-1 (Sigma-Aldrich, Merck KGaA, Darmstadt, Germany). To measure the inhibitory effect of AG on cold-induced RhoA activation, HUVECs and pericytes were pretreated with AG (100 or $200 \mu \mathrm{g} / \mathrm{ml}$ ) for $30 \mathrm{~min}$, followed by a warm $\left(37^{\circ} \mathrm{C}\right)$ or cold $\left(25^{\circ} \mathrm{C}\right)$ incubation for $30 \mathrm{~min}$. Cells were lysed for protein extraction using ice-cold radioimmunoprecipitation assay buffer containing $50 \mathrm{mM}$ Tris- $\mathrm{HCl}$, (pH 7.5), $150 \mathrm{mM} \mathrm{NaCl}$, $1 \%$ triton $\mathrm{X}-100,2 \mathrm{mM}$ EDTA, $0.1 \%$ SDS and $1 \%$ sodium deoxycholate (R2002; Biosesang Co., Ltd., Seoul, Korea). The extracted proteins were mixed with sample buffer (EBA-1052; Daejeon, Korea) and boiled at $100^{\circ} \mathrm{C}$ for $10 \mathrm{~min}$. Protein amount was analyzed by using Bio-Rad protein assay kit (500-0006; Bio-Rad GmbH, Munchen, Germany). Equal amount of proteins $(10 \mu \mathrm{g})$ were separated by $8-12 \%$ SDS-PAGE and transferred onto a nitrocellulose blotting membrane. The blots were probed with the following primary antibodies: Mouse-anti-active RhoA monoclonal (26904; 1:500; NewEast Biosciences, Malvern, PA, USA), rabbit-anti-phospho-focal adhesion kinase (FAK) monoclonal $(8556 ; 1: 500)$ and rabbit-anti-proto-oncogene tyrosine-protein kinase Src (SRC) polyclonal (2101; 1:1,000; both from Cell Signaling Technology, Inc., Danvers, MA, USA), mouse-anti-phospho-extracellular signal-related kinase (ERK) monoclonal (sc7383; $1: 1,000)$ and mouse-anti- $\beta$-actin monoclonal (sc73615; 1:1,000; both from Santa Cruz Biotechnology, Inc., Dallas, TX, USA). After blocking the membrane with $2 \%$ skim milk for non-phosphoform of proteins or $2 \%$ Bovine serum albumin (BSA) in TBST for phosphoform of proteins, primary antibodies were incubated with the membrane overnight at $4^{\circ} \mathrm{C}$ on a shaker. Secondary antibodies for mouse (7076; $1: 1,000-3,000)$ and rabbit $(074 ; 1: 1,000-3,000)$ were purchased from Cell Signaling Technology (Danvers, MA, USA) and incubated with the membrane for $1 \mathrm{~h}$ at room temperature on shaker. Antibody-conjugated membranes were incubated with ECL reagent (DG-WP250; DoGen, Seoul, Korea).

ELISA. ET-1 production in HUVEC-conditioned medium was evaluated using an endothelin-1 ELISA kit, according to the manufacturer's protocol (ADI-900-020A; Enzo Life Sciences, Inc., Farmingdale, NY, USA).

Phalloidin staining. The cold-induced formation of stress fibers and focal adhesion complexes was assessed in HUVECs. Cells were treated as aforementioned, and fixed with $4 \%$ paraformaldehyde (Junsei Chemical Co., Ltd., Tokyo, Japan) for $30 \mathrm{~min}$ and permeabilized with $0.1 \%$ Triton X-100 (T8787; Sigma-Aldrich, Merck KGaA, Darmstadt, Germany) for 15 min. Cells were stained with rhodamine-phalloidin (R415; Thermo Fisher Scientific, Inc., Waltham, MA, USA). The cells were visualized with Olympus FV10i self-contained confocal laser system (Olympus America Inc., PA, USA).

Statistical analysis. The differences of means between the groups were analyzed one-way analysis of variance. $\mathrm{P}<0.05$ was considered to indicate a statistically significant difference.

\section{Results}

$A G$ reduces cold- and ET-1-induced RhoA activation in pericytes. Pericytes contribute to microvascular contraction; therefore, the inhibitory effect of AG on the activity of RhoA in cold- or ET-1-exposed pericytes was examined by western blotting with active RhoA-GTP monoclonal antibody. AG treatment inhibited cold-induced RhoA activation (Fig. 1A), and ET-1-induced RhoA activation (Fig. 1B).

AG reduces cold-induced RhoA activation in HUVECs. The impact of AG treatment on cold-induced RhoA activation was investigated in HUVECs. AG treatment decreased cold-induced RhoA activation (Fig. 2). Therefore, AG treatment demonstrated an ability to inhibit cold-induced RhoA activation in both pericytes and HUVECs.

AG reduces cold-induced ET-1 production in HUVECs. ET-1 is produced by ECs and serves a key role in vasoconstriction under cold conditions (7-9). Therefore, the inhibitory effect of AG treatment on ET-1 production in cold-exposed ECs was examined. HUVECs exposed to cold for 30 min increased ET-1 production by approximately 2-fold compared with the cells under normal conditions $(\mathrm{P}<0.05)$, however, AG pretreatment did not impact on cold-induced ET-1 production (Fig. 3). 


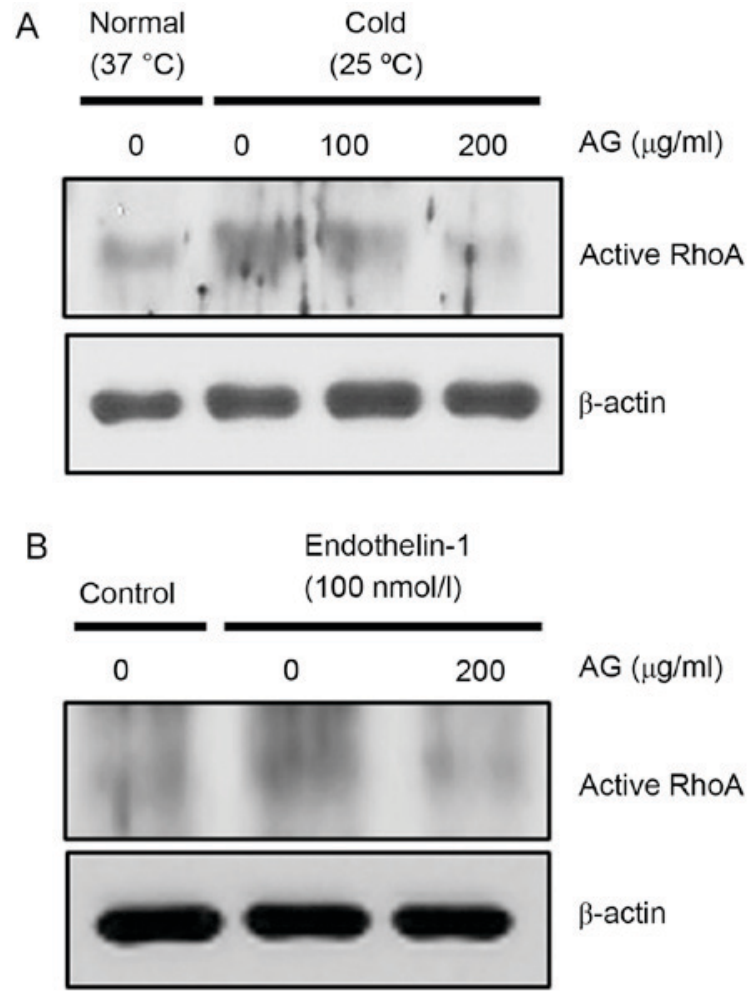

Figure 1. AG reduces cold- and endothelin-1-induced RhoA activation in pericytes. (A) Expression of active RhoA was measured in pericytes pre-treated with AG $(200 \mu \mathrm{g} / \mathrm{ml})$ for $30 \mathrm{~min}$, and then exposed to normal $\left(37^{\circ} \mathrm{C}\right)$ or cold $\left(25^{\circ} \mathrm{C}\right)$ temperatures. (B) RhoA expression was measured in pericytes pre-treated with $\mathrm{AG}(200 \mu \mathrm{g} / \mathrm{ml})$ for $30 \mathrm{~min}$, and then incubated with endothelin-1 (100 nM/l). $\beta$-actin served as the loading control. AG, Angelica gigas; RhoA, ras homolog gene family member A.

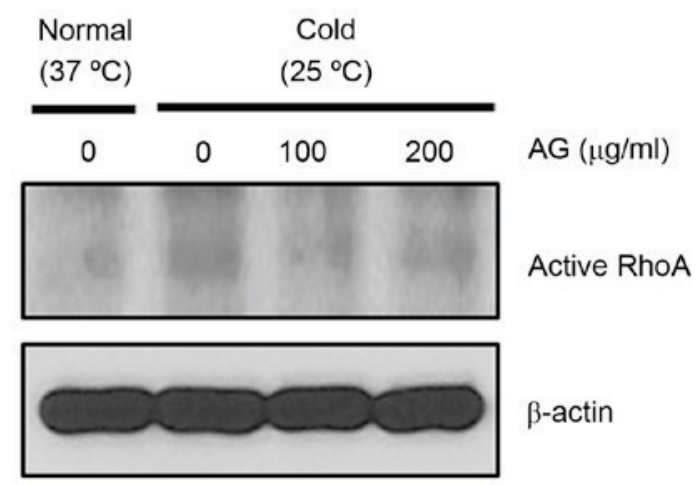

Figure 2. AG reduces cold-induced RhoA activation in HUVECs. HUVECs were pre-treated with $\mathrm{AG}(200 \mu \mathrm{g} / \mathrm{ml})$ for $30 \mathrm{~min}$ and then exposed to normal $\left(37^{\circ} \mathrm{C}\right)$ or cold $\left(25^{\circ} \mathrm{C}\right)$ temperatures. Expression of active RhoA was measured by western blot analysis, $\beta$-actin served as the loading control AG, Angelica gigas; RhoA, ras homolog gene family member A; HUVECs, human umbilical vein endothelial cells.

$A G$ reduces the cold-mediated formation of stress fibers and focal adhesion complexes in HUVECs. Cold-mediated RhoA activation induces the phosphorylation of FAK, which stimulates the formation of stress fibers and focal adhesion complexes (4). Cold exposure induced the formation of stress fibers and focal adhesion complexes, whereas AG treatment limited these cold-mediated responses (Fig. 4A). Furthermore, FAK phosphorylation stimulates the phosphorylation of SRC and ERK,

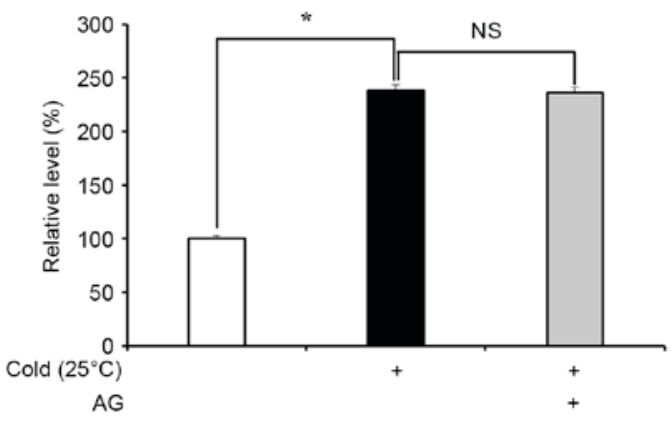

Figure 3. AG has no impact on cold-induced ET-1 production in HUVECs. HUVECs were pre-treated with $\mathrm{AG}(200 \mu \mathrm{g} / \mathrm{ml})$ for $30 \mathrm{~min}$ and then exposed to normal $\left(37^{\circ} \mathrm{C}\right)$ or cold $\left(25^{\circ} \mathrm{C}\right)$ temperatures for $30 \mathrm{~min}$. ET-1 was measured in cultured medium from HUVECs by ELISA. Experiments were performed in triplicate. Bars indicate the mean \pm standard deviation. ${ }^{*} \mathrm{P}<0.05$. AG, Angelica gigas; ET-1, endothelin-1; HUVECs, human umbilical vein endothelial cells; NS, not significant.

and western blot analysis demonstrated that the cold-induced phosphorylation of FAK, SRC and ERK was inhibited by AG treatment (Fig. 4B). Expression of unphosphorylated FAK, SRC and ERK was not changed (data not shown).

\section{Discussion}

AG has been used in the treatment vascular disorders, however its molecular mechanism remains unclear (18-21). The activation of RhoA may serve as an indicator for cold responses in vascular cells, however the cold-mediated mechanisms of RP remain to be elucidated (4-6). Our previous study demonstrated that cold-mediated contraction is tightly regulated by RhoA activity (4). In the present study, cold- and ET-1-induced RhoA activation in vascular cells was reduced by AG treatment. Furthermore, the data suggested that AG treatment may inhibit vascular cellular contraction via RhoA suppression, in HUVECs and pericytes. Although AG treatment did not affect cold-mediated ET-1 production in HUVECs, ET-1-mediated RhoA activation in pericytes was reduced, indicating that AG may demonstrate efficacy these cells. In addition, it may be hypothesized that cold-induced ET-1 production is likely to be regulated by a mechanism other than the RhoA-mediated pathway, suggesting that studies should be performed to elucidate the precise mechanism of cold-induced ET-1 production. However, further in vitro and in vivo experiments are necessary to elucidate which active compounds in $\mathrm{AG}$ are responsible for the observed RhoA-inhibitory effects. In addition, the results indicated that cold exposure may induce the formation of stress fibers and focal adhesion complexes, and these responses were reduced by AG treatment. Previous research has demonstrated that cell contraction and the formation of focal adhesions depend on RhoA signaling pathways $(23,24)$. The present study indicated that AG treatment was able to inhibit cold-mediated RhoA activation, thus resulting in a blockade of RhoA-mediated cell contraction and formation of focal adhesion complexes. Therefore, AG inhibition of RhoA activation may suppress vascular cellular contraction in cold conditions.

In conclusion, the present study demonstrated the inhibitory effect of AG on cold-induced contractile responses in vascular cells. Targeting RhoA by AG may be a useful therapeutic 


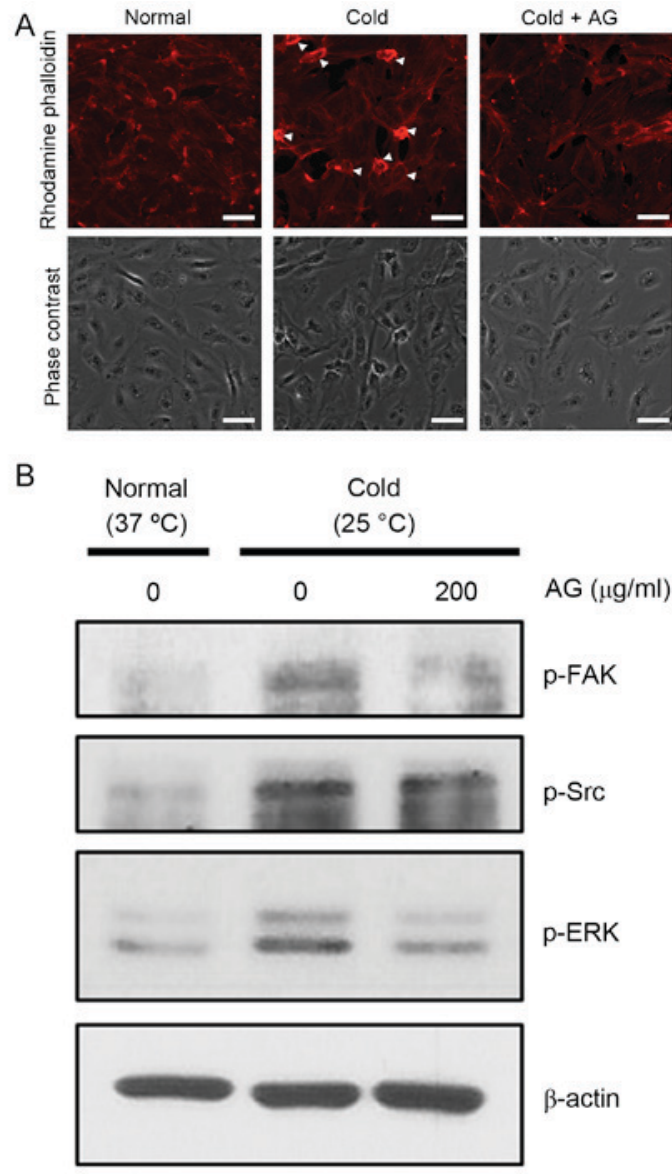

Figure 4. AG reduces the cold-mediated formation of stress fibers and focal adhesion complexes in HUVECs. (A) HUVECs were treated with AG $(200 \mu \mathrm{g} / \mathrm{ml})$ for $30 \mathrm{~min}$ and then exposed to normal $\left(37^{\circ} \mathrm{C}\right)$ or cold $\left(25^{\circ} \mathrm{C}\right)$ temperatures for $30 \mathrm{~min}$. F-actin was detected by rhodamine-phalloidin staining. The experiment was performed triplicate, and three fields were imaged in each treatment group. Figures are of a representative image. Arrowheads indicate the formation of stress fibers and focal adhesion complexes (scale bar=50 $\mu \mathrm{m}$ ). (B) Phosphorylated forms of FAK, SRC, and ERK were detected by western blot analysis, $\beta$-actin served as the loading control. AG, Angelica gigas; HUVECs, human umbilical vein endothelial cells; FAK, focal adhesion kinase; SRC, proto-oncogene tyrosine-protein kinase Src; ERK, extracellular signal-related kinase.

strategy for the treatment of vascular diseases, including RP. Further in vitro and in vivo studies are required, and these should investigate the vasodilatory impact of AG, and elucidate the active compound responsible for these effects.

\section{Acknowledgements}

Human umbilical vein endothelial cells (HUVECs) were gifted by Kwang Seok Kim at Korea Institute of Radiological and Medical Science (Seoul, Korea). This study was supported by the Korean Medicine R\&D Project of the Ministry of Health and Welfare (grant no. HI13C0530).

\section{References}

1. Cooke JP and Marshall JM: Mechanisms of Raynaud's disease Vasc Med 10: 293-307, 2005.

2. Herrick AL: Pathogenesis of Raynaud's phenomenon. Rheumatology (Oxford) 44: 587-596, 2005.

3. LeRoy EC and Medsger TA Jr: Raynaud's phenomenon: A proposal for classification. Clin Exp Rheumatol 10: 485-488, 1992.
4. Cho SG, Go HY, Park JS, Jung KY, Sun SH, Choi YK, Song YK, Park JH, Jun CY and Ko SG: Herbal prescription, DSGOST, prevents cold-induced RhoA activation and endothelin-1 production in endothelial Cells. Evid Based Complement Alternat Med 2014: 549307, 2014.

5. Thompson-Torgerson CS, Holowatz LA, Flavahan NA and Kenney WL: Cold-induced cutaneous vasoconstriction is mediated by rho kinase in vivo in human skin. Am J Physiol Heart Circ Physiol 292: H1700-H1705, 2007.

6. Bailey SR, Eid AH, Mitra S, Flavahan S and Flavahan NA: Rho kinase mediates cold-induced constriction of cutaneous arteries: Role of alpha2C-adrenoceptor translocation. Circ Res 94: 1367-1374, 2004.

7. Rychlik-Golema W, Mastej K and Adamiec R: The role of endothelin-1 and selected cytokines in the pathogenesis of Raynaud's phenomenon associated with systemic connective tissue diseases. Int Angiol 25: 221-227, 2006.

8. Zamora MR, O'Brien RF, Rutherford RB and Weil JV: Serum endothelin-1 concentrations and cold provocation in primary Raynaud's phenomenon. Lancet 336: 1144-1147, 1990.

9. Barman SA: Vasoconstrictor effect of endothelin-1 on hypertensive pulmonary arterial smooth muscle involves Rho-kinase and protein kinase C. Am J Physiol Lung Cell Mol Physiol 293: L472-L479, 2007.

10. Sakurada S, Okamoto H, Takuwa N, Sugimoto N and Takuwa Y: Rho activation in excitatory agonist-stimulated vascular smooth muscle. Am J Physiol Cell Physiol 281: C571-C578, 2001.

11. Dehouck MP, Vigne P, Torpier G, Breittmayer JP, Cecchelli R and Frelin C: Endothelin-1 as a mediator of endothelial cell-pericyte interactions in bovine brain capillaries. J Cereb Blood Flow Metab 17: 464-469, 1997.

12. Wu YJ, Luo SF, Yang SH, Chen JY, Yu KH and See LC: Vascular response of Raynaud's phenomenon to nifedipine or herbal medication (duhuo-tisheng tang with danggui-sini tang): A preliminary study. Chang Gung Med J 31: 492-502, 2008.

13. Malenfant D, Catton $M$ and Pope JE: The efficacy of complementary and alternative medicine in the treatment of Raynaud's phenomenon: A literature review and meta-analysis. Rheumatology (Oxford) 48: 791-795, 2009.

14. Ninomiya F: Clinical evaluation of perspiration reducing effects of a kampo formula, shigyaku-san, on palmoplantar hidrosis. Evid Based Complement Alternat Med 5: 199-203, 2008.

15. Kanai S, Okano H and Abe H: Efficacy of toki-shigyakukagosyuyu-syokyoto (danggui-sini-jia-wuzhuyu-shengjiang-tang) on peripheral circulation in autonomic disorders. Am J Chinese Med 25: 69-78, 1997.

16. Park KS, Park KI, Kim JW, Yun YJ, Kim SH, Lee CH, Park JW and Lee JM: Efficacy and safety of Korean red ginseng for cold hypersensitivity in the hands and feet: A randomized, double-blind, placebo-controlled trial. J Ethnopharmacol $158 \mathrm{Pt}$ A: 25-32, 2014

17. Choi KO, Lee I, Paik SY, Kim DE, Lim JD, Kang WS and Ko S: Ultrafine Angelica gigas powder normalizes ovarian hormone levels and has antiosteoporosis properties in ovariectomized rats: Particle size effect. J Med Food 15: 863-872, 2012.

18. Kim KM, Kim MJ and Kang JS: Absorption, distribution, metabolism, and excretion of decursin and decursinol angelate from Angelica gigas Nakai. J Microbiol Biotechnol 19: 1569-1572, 2009.

19. Jang JY, Kim J, Cai J, Kim Y, Shin K, Kim TS, Lee SP, Park SK, Choi EK and Kim YB: An ethanolic extract of Angelica gigas improves atherosclerosis by inhibiting vascular smooth muscle cell proliferation. Lab Anim Res 30: 84-89, 2014.

20. Oh TW, Park KH, Jung HW and Park YK: Neuroprotective effect of the hairy root extract of Angelica gigas NAKAI on transient focal cerebral ischemia in rats through the regulation of angiogenesis. BMC Complement Altern Med 15: 101, 2015.

21. Rhyu MR, Kim EY, Yoon BK, Lee YJ and Chen SN: Aqueous extract of Schizandra chinensis fruit causes endothelium-dependent and -independent relaxation of isolated rat thoracic aorta. Phytomedicine 13: 651-657, 2006.

22. Rhyu MR, Kim JH and Kim EY: Radix Angelica elicits both nitric oxide-dependent and calcium influx-mediated relaxation in rat aorta. J Cardiovasc Pharmacol 46: 99-104, 2005.

23. Yao L, Romero MJ, Toque HA, Yang G, Caldwell RB and Caldwell RW: The role of RhoA/Rho kinase pathway in endothelial dysfunction. J Cardiovasc Dis Res 1: 165-170, 2010.

24. van Nieuw Amerongen GP, Koolwijk P, Versteilen A and van Hinsbergh VW: Involvement of RhoA/Rho kinase signaling in VEGF-induced endothelial cell migration and angiogenesis in vitro. Arterioscler Thromb Vasc Biol 23: 211-217, 2003. 